\title{
Outsurvival as a measure of the inequality of lifespans between two populations
}

\author{
by James W. Vaupel, Marie-Pier Bergeron-Boucher and Ilya Kashnitsky \\ Danish Center for Demographic Research and Interdisciplinary Center on Population Dynamics, \\ University of Southern Denmark, Odense, Denmark
}

\begin{abstract}
Background: Inequality in lifespans between two populations, e.g., males and females or people with low and high SES, is a focus of demographic, economic and sociological research and of public policy analysis. Inequality is usually measured by differences in life expectancy.

Objective: To devise a cogent measure of how much distributions of lifespans differ between two populations.

Results: We propose an outsurvival statistic, $\varphi$, that measures the probability that an individual from a population with low life expectancy will live longer than an individual from a population with high life expectancy. This statistic can also be interpreted as an underdog probability - the chance that a random value from a distribution with a low mean will exceed a random value from a distribution with a higher mean.

Contribution: Our outsurvival probability complements life expectancy to provide a more nuanced view of the inequality of lifespans between two populations. Our similar underdog probability provides a perspective on the more general question of how different two distributions are.
\end{abstract}

Keywords: Lifespan inequalities, population distributions, comparing two distributions 


\section{Relationship}

Consider two populations. Suppose ages at death (lifespans) in the first population are independent of ages at death in the second population. Let $\mu_{i}(x), i=1,2$, denote the force of mortality at age $x$ in two populations, which could be males and females or people with low and high SES. Assume that the first population has the lower life expectancy. Let $\ell_{i}(x)$ denote survival over age and let $d_{i}(x)=$ $\mu_{i}(x) \ell_{i}(x)$, denote the density of ages at death, i.e. lifespans. Consider two randomly selected individuals or, equivalently, all possible pairs of individuals, one from each population, at the starting age, which could be birth or, say, age 65 . The probability the individual from the first population will outlive the individual from the second population is:

$$
\varphi=\int_{0}^{\infty} d_{2}(x) \ell_{1}(x) d x=\int_{0}^{\infty} \mu_{2}(x) \ell_{2}(x) \ell_{1}(x) d x .
$$

As described later, there are various measures of the difference between two distributions. Use of eq. (1) to shed light on lifespan inequality between two populations is, to our knowledge, new. We developed the measure $\varphi$ rather than relying on some other approach because of our interest in how often individuals in one group live longer than individuals in another group. Hence, eq. (1) pertains to the distribution of lifespans in two populations. A lifespan has a start (e.g., birth or attaining some age or the start of use of some equipment such as an automobile or a lightbulb) and an end (e.g., death or the failure of the equipment). Usually lifespans are measured by the duration between birth and death, but the lifespan of an automobile could be summarized by kilometers driven, the lifespan of a rabbit by number of offspring, the lifespan of a worker by total wages, and the lifespan of a scholar by articles published or citations received.

Proof of (1) is straightforward. In the first variant of the formula, the value of $d_{2}(x)$ gives the probability that the lifespan of an individual from population 2 is $x$ and $\ell_{1}(x)$ is the probability that the lifespan of an individual from population 1 is greater than $x$. Hence the product is the chance that 
the individual from the first population will outsurvive the individual from the second population. Summing the products over age gives the overall probability for all individuals.

\section{Related results}

Note that

$$
1-\varphi=\int_{0}^{\infty} \mu_{1}(x) \ell_{1}(x) \ell_{2}(x) d x
$$

so that

$$
\int_{0}^{\infty}\left(\mu_{1}(x)+\mu_{2}(x)\right) \ell_{1}(x) \ell_{2}(x) d x=1 .
$$

If the two populations have the same age-patterns of mortality, (1) leads to

$$
\varphi=\int_{0}^{\infty} d(x) \ell(x) d x=\int_{0}^{\infty} \mu(x) \ell^{2}(x) d x=0.5
$$

The probability $\varphi$ is zero if the longest lifespan in the first population is less than the shortest lifespan in the second population. The value of 0.5 is not the maximum possible value of $\varphi$. Consider an extreme case when everyone in the first population dies close to the same age, $X_{1}$, so life expectancy for this population is $X_{1}$. Suppose for the second population, $99 \%$ of the population dies at birth, but the remaining $1 \%$ survive to an age $X_{2}>100 X_{1}$. The life expectancy of the second population, $0.01 X_{2}$ exceeds $X_{1}$, but $\varphi$ is 0.99 . As a second example, suppose that for $99 \%$ of the first population the age at death is close to a value $X_{1}$ but $1 \%$ die at birth. Life expectancy is $0.99 X_{1}$. In contrast, suppose for the second population that everyone has a lifespan close to $X_{2}$ but less than $X_{1}$ with $0.99 X_{1}<X_{2}<X_{1}$. Again $\varphi$ is 0.99 . More generally, it can be seen that $0 \leq \varphi<1$.

Suppose the two populations have constant forces of mortality over age, $\mu_{1}(x)=\mu$ and $\mu_{2}(x)=$ $\mu / 2$. Then life expectancy for the first population, given by $1 / \mu$, is half that life expectancy of the second population. Because

$$
\varphi=\int_{0}^{\infty} \mu_{2} e^{-\mu_{2} x} e^{-\mu_{1} x} d x=\frac{\mu_{2}}{\mu_{1}+\mu_{2}}
$$


the value of $\varphi$ is $1 / 3$. Despite the large difference in life expectancy, an individual in the first population has one chance in three of outliving a contemporary in the second population.

Instead of pertaining to two individuals from two populations, eq. (1) can be used to analyze two independent competing risks of death for an individual. Then $\varphi$ gives the probability of death from cause 2 rather than cause 1 . The equation makes it clear that to die from cause 2 , a person has to survive death from cause 1, i.e., from all hazards except cause 2. This is well known but sometimes forgotten. The chance that a person from some population will outlive a person from another population is conceptually different from the chance a person dies from some cause rather than another cause, but $\varphi$ is an appropriate measure in both cases.

Eq. (1) is readily generalized to discrete distributions (Bergeron-Boucher et al., 2020).

\section{Applications}

Stratification has stimulated considerable interest - how distinct are two populations based on the distribution of some characteristic capturing, e.g., social status or political or religious views (Shi et al., 2020; Zhou \& Wodtke, 2019)? Fig. 1 provides an illustration that uses lifespan data. The distributions were estimated from lifetables for French males and females age 65 in 2018. One measure of the stratification of the two distributions is the overlapping index (Gini \& Livada, 1943; Pastore \& Calcagnì, 2019), the area coloured purple, which is given by

$$
\eta=\int_{0}^{\infty} \min \left(d_{1}(x), d_{2}(x)\right) d x
$$

In Fig. 1, the overlapping area $\eta$ is 0.81 . Because the area under the density distribution of deaths is one, the combined area of the non-overlapping distributions, coloured red and blue, is $2(1-\eta)$. The red area is actually the same as the blue area, $(1-\eta)$. The proportion of the non-overlapping areas to the total area, i.e., the ratio of the sum of the areas coloured red and blue to the sum of those two areas and the common area coloured purple, is $2(1-\eta) /(2-\eta)$ and has a value of 0.32 in Fig. 1; this statistic 
was used by Shi et al. (2020). Interpretation of measures of overlap is often difficult and the meaning of such measures may not be clear to policy analysts studying inequality.

Figure 1. Lifetable age at death distributions from age 65 for French males and females, 2018. The ratio of the blue plus red areas to the total area is $32 \%$.

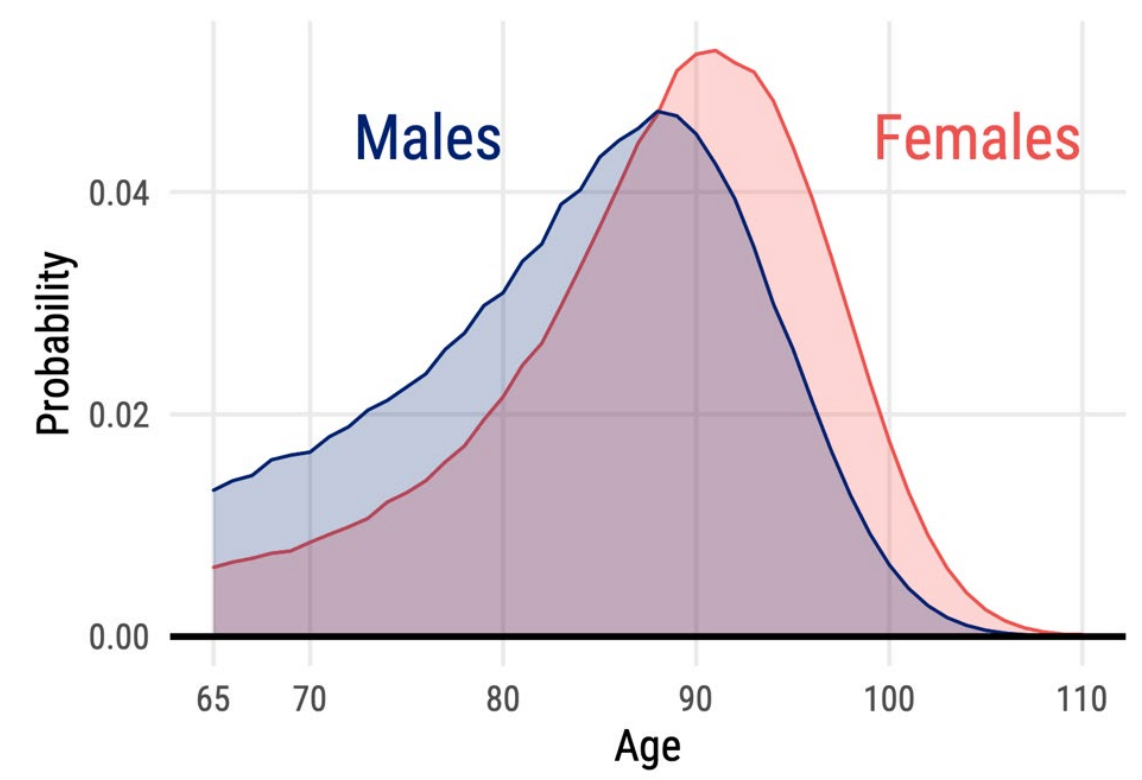

Source: Own calculations applied to the HMD (2020) French life tables by sex for 2018.

Our approach, as given by the first formula in eq. (1), is illustrated in Fig. 2, using the same French data used in Fig. 1. Fig. 2a displays $\ell_{1}(x)$, the survival curve for the French males. Fig. 2b shows $d_{2}(x)$, the distribution of ages at death for the French females, the population with higher life expectancy. The area shaded in green in Fig. $2 \mathrm{~b}$ is the area defined by the product of the $\ell_{1}(x)$ and $d_{2}(x)$ curves: this area, which equals $\varphi$, is 0.37 . Hence, although remaining life expectancy at age 65 was 19.47 for French males, almost four years less than the value of 23.39 for French females, $37 \%$ 
of the males would outlive a corresponding female ${ }^{1}$. Note that because death is inevitable, the area under the $d_{2}$ curve is 1 . Hence the grey area in Fig. 2 is $1-0.37$ or 0.63 .

Figure 2. Graphical representation of $\varphi$ : A - Survival curve of French males; B - life table age at death distribution of French females and the product of female age at death distribution and male survival (green area).
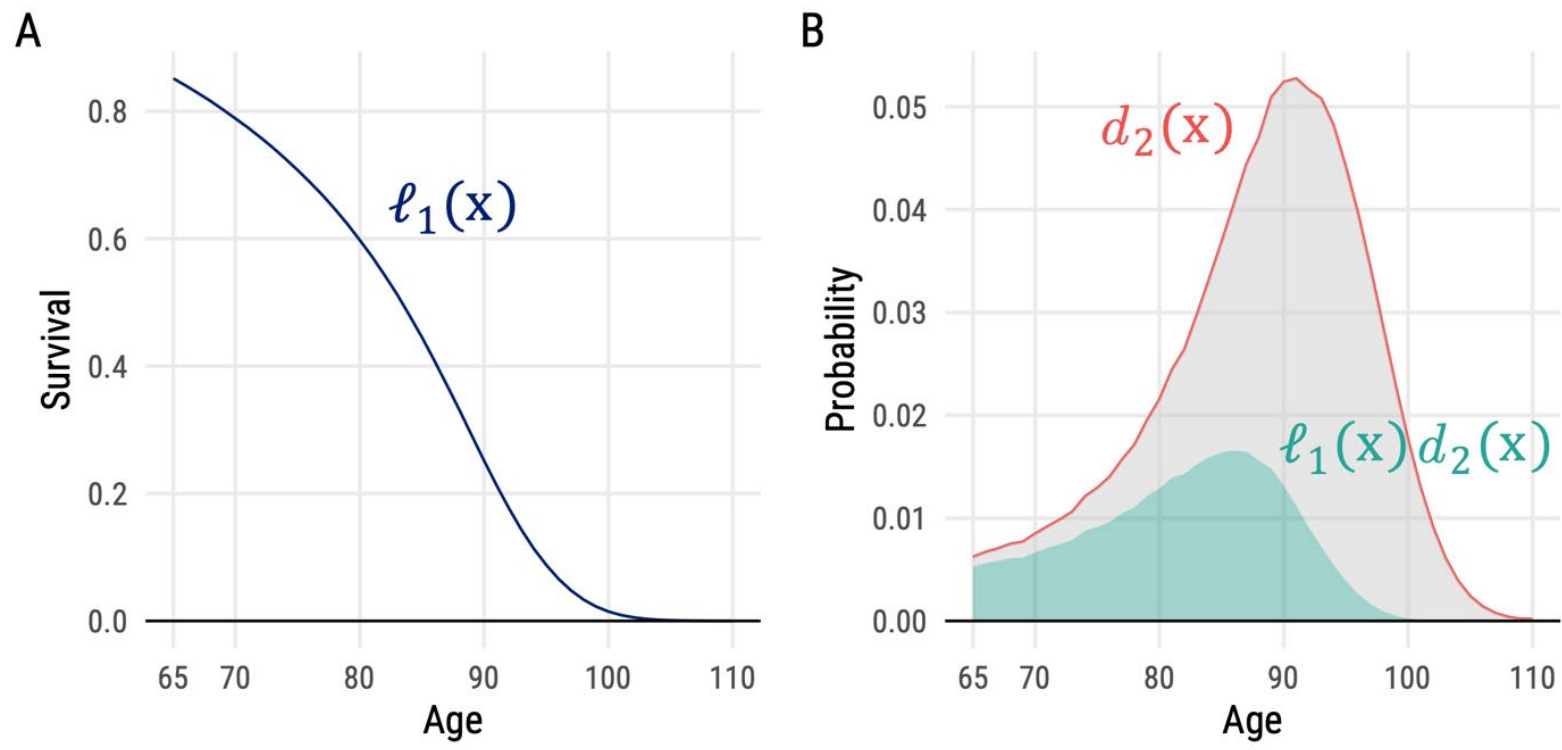

Source: Own calculations using the discrete approximations defined in Bergeron-Boucher et al. (2020) applied to the HMD (2020) French life tables by sex for 2018.

Lifetables for the United States in 2017 (CDC, 2019) show that non-hispanic blacks had a life expectancy at birth of 74.9 years compared with 78.5 years for non-hispanic whites. Yet $\varphi$ was 0.439 : in nearly $44 \%$ of possible pairs, the non-hispanic black would outlive the non-hispanic white ${ }^{2}$.

\footnotetext{
${ }^{1}$ The value of $\varphi$ was estimated using the discrete approximation defined in Bergeron-Boucher et al. (2020) applied to the HMD (2020) life tables by sex. Two other ways to estimate $\varphi$ were analyzed By Bergeron-Boucher et al. (2020): (1) random pairing of individual lifespans, based on simulated individual lifespans from empirical death rates and (2) continuous approximation using eq. (1) based on simulated lifespan distributions. All three methods provided equivalent results.

${ }^{2}$ The value of $\varphi$ was estimated using the discrete approximation as defined in Bergeron-Boucher et al. (2020) applied to the CDC (2019) life tables by race.
} 
Lifetables by education level in Italy for the year 2012 (ISTAT, 2020) show that, at age 30, men with a university degree had a remaining life expectancy of 53.1 years, while men with lower secondary education had a remaining life expectancy of 50.2 years. Our computations indicate that $\varphi$ was $0.431^{3}$.

\section{Directions for further research}

Our measure $\varphi$ of between-population lifespan inequality could be applied to many pairs of male and female lifetables to study how inequality differs across populations and is changing over time. Such analysis has recently been completed (Bergeron-Boucher et al., 2020). There are many other possible applications, to nonhuman and nonliving populations as well as human populations. For example, a major concern is inequality among SES groups in how long people live after retirement.

How does life expectancy vary with perturbations in mortality? This is a question that has led to some informative mathematical relationships (Vaupel, 1986; Wrycza \& Baudisch, 2012). A similar question can be asked — how does outsurvival, $\varphi$, vary with perturbations in mortality? In particular, how much would lifespan inequality between two populations be reduced or increased by different patterns of change in age-specific mortality and resulting life expectancy? As a simple example, consider eq. (5), which implies

$$
\varphi=\frac{1}{1+\frac{\mu_{1}}{\mu_{2}}}=\frac{1}{1+\frac{e_{2}}{e_{1}}}
$$

where $\mathrm{e}_{\mathrm{i}}$ denotes life expectancy. If the ratio of life expectancies is 2 , then outsurvival is a third, but if the ratio is 3 , then $\varphi$ worsens to 0.25 .

\footnotetext{
${ }^{3}$ The value of $\varphi$ was estimated using the discrete approximation as defined in Bergeron-Boucher et al. (2020) based on the ISTAT (2020) life tables by education group.
} 
The specific value of $\varphi$ depends on the difference between the life expectancies of the two population and also on the shape of the distributions. It would be interesting to derive some formal relationships.

Also interesting for future research would be to focus on the cases when an individual from the first population outlives an individual from the second population - to work out outsurvival time, i.e., how much longer the first individual lives.

Calculations of $\varphi$ assume that the two lifespan distributions are independent. This is often not true for matched individuals, e.g., spouses or twins. More generally, a complicated problem that merits further study is how joint probability density functions should be estimated for correlated lifespans of pairs of individuals.

\section{Extension}

Let $f_{1}(x)$ and $f_{2}(x)$ be two probability density functions and let $F_{1}(x)$ and $F_{2}(x)$ be the corresponding cumulative distributions. Then,

$$
\varphi=\int_{0}^{\infty} f_{1}(x) F_{2}(x) d x
$$

This corresponds to eq. (1) with $f_{1}(x) \equiv d_{1}(x)$ and $F_{2}(x)=\int_{0}^{x} d_{2}(t) d t$ because

$$
\begin{aligned}
\varphi=\int_{0}^{\infty} d_{2}(x) & \ell_{1}(x) d x \\
& =\int_{0}^{\infty} d_{2}(x) \int_{x}^{\infty} d_{1}(t) d t d x \\
& =\int_{0}^{\infty} d_{1}(x) \int_{0}^{x} d_{2}(t) d t d x=\int_{0}^{x} f_{1}(x) F_{2}(x) d x .
\end{aligned}
$$

Eq. (1) and (8) are equivalent when studying lifespan inequalities. Bergeron-Boucher et al. (2020) also show this equivalency and how eq. (1) relates to the joint probability density function. 
If in eq. (8), the first density distribution has a lower mean than the second density distribution, then $\varphi$ could be called the underdog probability — the chance that an individual or item from the distribution with the lower mean turns out to be bigger or greater than the corresponding value from the distribution with the higher mean. Calculation of underdog probabilities can be applied to a wide range of topics. For example, it can be used to study income inequality between sexes. According to the US Census Bureau, the mean income for females was $\$ 43,315$, while it was $\$ 65,144$ for males in 2019 (U.S. Census Bureau, 2020). Using eq. (8), we can estimate that the probability of females having a higher salary than males is $38.5 \%{ }^{4}$.

As a second example, consider the ages of people in Italy and Ireland. In 2019 the median age in Italy was 46.2 whereas the median age in Ireland was 37.2 (EUROSTAT, 2020). If a random person from Ireland was matched with a random Italian, in two out of five pairs the Irish person would be older ${ }^{5}$.

A related research question was recently studied in bibliometrics (Brito \& Rodríguez-Navarro, 2019). The authors challenged the commonly used practice of evaluating an article based on the Impact Factor of the journal in which it is published. In the experiment, randomly chosen articles from a pair of journals were compared based on their journals' Impact Factors and the actual citations gained in the recent years. Via extensive random paring the researchers estimated the proportion of cases when the article in the journal with the higher Impact Factor was the article that was more cited. This turned out to be close to $50 \%$, like flipping a coin, indicating that the common strategy of proxying an academic paper's quality with the Impact Factor of the journal is problematic.

\footnotetext{
${ }^{4}$ The value of $\varphi$ was estimated using the discrete approximation defined in Bergeron-Boucher et al. (2020) applied to the U.S. Census Bureau (2020) income distribution data by sex.

${ }^{5}$ The value of $\varphi$ was estimated using the discrete approximation defined in Bergeron-Boucher et al. (2020) applied to EUROSTAT (2020) population distribution data by age for Italy and Ireland.
} 


\section{Discussion}

How much two density distributions differ is a topic of a vast literature that includes overlapping indices as well as calculations of within and between group variance. One major line of research is the development and application of approaches to determine how large the vertical difference is between two distributions according to measures of statistical distance: two examples are KullbackLeibler divergence and Kolmogorov distance. These and various measures of horizontal differences (the earth mover's problem) have been applied to distributions of lifespans, income, IQ, temperature, etc. (D’ambrosio, 2001; Deza \& Deza, 2016; Edwards \& Tuljapurkar, 2005; Naveau et al., 2014; Permanyer \& Scholl, 2019).

Our measure $\varphi$ is a probability that sheds light on a different question: how distinct are two density distributions? If the mean of one distribution is bigger than the mean of the other, it is sometimes claimed this holds for all individuals. For example, "the life expectancy of men is shorter than that of women" is often sloppily summarized as "men do not live as long as women". Let $X_{1}$ be the remaining lifespan of a man and let $X_{2}$ be the remaining lifespan of a woman of the same age. Then the probability that $X_{1}>X_{2}$ is $\varphi$. More generally, $\varphi$ captures the probabilistic correctness of the assertion that individuals from a distribution with a low mean have values less than those of individuals from a distribution with a higher mean. Probabilistic correctness is a different concept than within vs. between group variance, the overlap of two density distributions, or the statistical distance according to some metric between two distributions. Eq. (1) and (8) are not difficult to prove and have been used in various contexts involving when it is of interest to estimate the chance that a random variable from one distribution exceeds a random variable from another distribution. For instance, the first author of this Formal Relationship studied eq. (8) as a graduate student in 1969 when he was learning about game theory: if two opponents have different uncertain strengths, what is the chance the first is stronger? Another example comes from reliability theory: what is the risk that some uncertain stress 
on some equipment will exceed the uncertain strength of the equipment (An et al., 2008)? In sum, the contribution made here is the application of straightforward mathematics in an innovative way to important questions in demography.

\section{References}

An, Z.-W., Huang, H.-Z., \& Liu, Y. (2008). A discrete stress-strength interference model based on universal generating function. Reliability Engineering \& System Safety, 93(10), 1485-1490.

Bergeron-Boucher, M.-P., Alvarez, J.-A., Kashnitsky, I., Zarulli, V., \& Vaupel, J. W. (2020, October 20). Not all females outlive all males: A new perspective on lifespan inequalities between sexes. SocArXiv, DOI: 10.31235/osf.io/typws.

Brito, R., \& Rodríguez-Navarro, A. (2019). Evaluating research and researchers by the journal impact factor: Is it better than coin flipping? Journal of Informetrics, 13(1), 314-324.

CDC. (2019). United States Life Tables, 2017. National Vital Statistics Report, 68(7).

D'ambrosio, C. (2001). Household characteristics and the distribution of income in Italy: an application of social distance measures. Review of Income and Wealth, 47(1), 43-64.

Deza, M., \& Deza, E. (2016). Encyclopedia of Distances, 4-th ed: Springer-Verlag.

Edwards, R. D., \& Tuljapurkar, S. (2005). Inequality in life spans and a new perspective on mortality convergence across industrialized countries. Population and Development Review, $31(4), 645-674$.

EUROSTAT. (2020). Population on 1 January by age and sex. Available at http://appsso.eurostat.ec.europa.eu/nui/show.do?wai=true\&dataset=demo pjan (Accessed on October 23).

Gini, C., \& Livada, G. (1943). Nuovi contributi alla teoria della transvariazione. Atti della VI Riunione della Societa Italiana di Statistica. Rome. 
HMD. (2020). Human Mortality Database. University of California, Berkeley (USA), and Max Planck Institute for Demographic Research (Germany), Available at www.mortality.org (Accessed on September 9) .

ISTAT. (2020). Life tables by educational attainment for the year 2012. Available at http://dati.istat.it/Index.aspx?DataSetCode=DCIS MORTALITA1\&Lang=en (Accessed on September 8).

Naveau, P., Guillou, A., \& Rietsch, T. (2014). A non-parametric entropy-based approach to detect changes in climate extremes. Journal of the Royal Statistical Society: Series B: Statistical Methodology, 861-884.

Pastore, M., \& Calcagnì, A. (2019). Measuring distribution similarities between samples: A distribution-free overlapping index. Frontiers in Psychology, 10, 1089.

Permanyer, I., \& Scholl, N. (2019). Global trends in lifespan inequality: 1950-2015. PloS one, 14(5), e0215742.

Shi, J., Aburto, J. M., Martikainen, P., Tarkiainen, L., \& van Raalte, A. A. (2020). Beyond differences in means: Rising mortality stratification among income groups in Finland, 19962014. Paper presented at the European Population Conference (EPC 2020), Padova, Italy. http://epc2020.popconf.org/sessions/9

U.S. Census Bureau. (2020). PINC-01. Selected Characteristics of People 15 Years and Over, by Total Money Income, Work Experience, Race, Hispanic Origin, and Sex. 2019 Total Work Experience. Available at https://www.census.gov/data/tables/time-series/demo/incomepoverty/cps-pinc/pinc-01.html (Accessed on October 18 2020).

Vaupel, J. W. (1986). How change in age-specific mortality affects life expectancy. Population Studies, 40(1), 147-157. 
Wrycza, T. F., \& Baudisch, A. (2012). How life expectancy varies with perturbations in agespecific mortality. Demographic Research, 27, 365-376.

Zhou, X., \& Wodtke, G. T. (2019). Income stratification among occupational classes in the United States. Social Forces, 97(3), 945-972. 